\title{
DETERMINING AGGREGATE SIZE \& SHAPE EFFECT ON CONCRETE MICROCRACKING UNDER COMPRESSION BY MEANS OF A DEGREE OF REVERSIBILITY METHOD
}

\author{
M. TOKYAY ${ }^{1} \&$ T. AKCAOGLU ${ }^{2}$ \\ ${ }^{1}$ Civil Engineering Department, Middle East Technical University, Ankara, Turkey \\ ${ }^{2}$ School of Computing and Technology, Eastern Mediterranean University, Famagusta, N. Cyprus
}

\begin{abstract}
It is an established fact that Interfacial Transition Zone (ITZ) between aggregate and mortar matrix, is the weakest area in the concrete composites. Crack initiation and propagation along the ITZ depends on the stress concentration in the ITZ and also to the matrix quality surrounding it. Under uniaxial compression tensile strains are produced in the transversal directions creating cracks along the axial direction. Thus, evaluation of the degree of reversibility (degree of reversible strain) with the level of loading determines the extent of microcracking (permanent damage) within the material at a certain loading level. The recorded strains provide an indirect method for understanding and quantifying the general relationship between stress level and crack development. In this study the effect of the aggregate size and shape and also the mortar matrix quality on the degree of reversibility were evaluated by subjecting the specimens to three different compressive stress levels $\left(40,60\right.$ and $80 \%$ of $\sigma_{\mathrm{c}}$ ). Experiments were designed and carried out on mortars with two different $\mathrm{w} / \mathrm{c}$ ratios containing single spherical, cylindrical and prismatic steel aggregates of different sizes. The degree of mechanical interlocking and internal stress concentration around the aggregate, related to the aggregate surface characteristics, has a significant influence on microcracking behaviour of concrete. Furthermore, aggregate surface characteristics become more pronounced with increased matrix quality.
\end{abstract}

Keywords: Aggregate, Matrix, Interfacial Transition Zone, Microcracking and Degree of Reversibility

\section{INTRODUCTION}

The mechanical properties and fracture behavior of concrete depends on the properties of the individual phases and their interactions. Cracks initiate at the interface between aggregate and the matrix. With increasing stress, matrix cracks forms and they gradually spread until they join the cracks originating from the interfacial transition zone (ITZ). The crack system then becomes continuous and the material ruptures (Shah [1]). Mitsuru [2] observed that, the rate of propagation of cracks along the interface varied with the aggregate properties. Similarly Jones [3], Chiaia [4], Prokopski [5] concluded that, the stress at which cracks develop largely depends on the properties of coarse aggregate. They mentioned that the relatively smooth and rounded shape of the aggregate provides a weak obstacle for crack initiation and propagation.

One of the ways of observing the occurred damage in the material is to measure the maximal and residual strains from loading-unloading hysteresis loops in any direction as proposed by Bascoul [6]. Thus, the damage occurred along either the longitudinal or transverse direction, under different loading stages can be deduced from strain measurements. Ringot [7] compared microcracking results obtained from degree of reversibility measurements along the axial direction with microcrack density obtained from microscopic analyses. He concluded that there is a linear relationship between the degree of reversibility and the microcrack density of concrete.

In the present study, a series of experiments were carried out to understand and quantify the general dependency of crack initiation and propagation on aggregate properties. The effect of the aggregate size and shape and also the mortar matrix quality on the degree of reversibility for the specimens subjected to three different compressive stress levels were evaluated. The surface characteristics of aggregates were found to be influential on microcracking behaviour of concrete. 


\section{THEORETICAL FOUNDATIONS AND EXPERIMENTAL PROCEDURE}

The evaluation of the degree of reversibility (\% Reversibility) with the level of loading characterizes the development of permanent damage in the material. In other words, the degree of reversible strain at different loading stages determines the extent of microcracking within the material at a certain loading level. \% Reversibility can be defined as proposed by Bascoul [6] as

$$
\% \text { Reversibility }=\left(\frac{\varepsilon_{t}-\varepsilon_{r}}{\varepsilon_{t}}\right) \times 100
$$

Where $\varepsilon_{t}$ and $\varepsilon_{r}$ are the maximum and residual strains measured in axial direction, respectively.

In this study both the size and shape of the aggregates effect and of the matrix qualities, on microcracking behavior of concrete composites, was studied by $\%$ Reversibility measurements. Experiments were carried out by two sets of mortar matrices $(w / c=0.82,0.42)$ containing single steel aggregates (spherical (S), cylindrical (C) and prismatic (P)) of different sizes. The strains recorded in these tests were in the longitudinal direction and at three loading levels (40,60 and $80 \%$ of compressive strength). It should be noted that in this experimental study the loading direction is perpendicular to the long side of the aggregate placed at the center of the $10 \mathrm{~cm}$ cube specimen. The results of the experiments are discussed below.

\section{TEST RESULTS AND DISCUSSIONS: AGGREGATE SIZE AND SHAPE EFFECT ON \% REVERSIBILITY}

Crack initiation and propagation along the ITZ depends on the stress concentration in the ITZ and also on the matrix quality surrounding it. The equidimensionality, angularity and sharp edges of the aggregates influences the mechanical bond between the aggregate and the matrix and also the stress concentration and distribution around the aggregate.

In Figs (1) and (2), the \% Reversibility measurements are plotted indicating the effect of different aggregate shapes for low strength composites (LSC) and high strength composites (HSC) respectively. In LSC \% Reversibility measurements are reasonably close to each other for a wide range of Surface Area/Volume (SA/V) of aggregates (at around 0.6-0.7). For the prismatic shape $\%$ Reversibility increases slightly with increasing SA/V. For the cylindrical shape on the other hand \% Reversibility decreases slightly with increased SA/V. The behavior of spherical shape is like the prismatic shape. However \% Reversibility of the spherical shape is lower both than the cylindrical and prismatic shape at all loading levels. In an opposite manner to the LSC the \% Reversibility of cylindrical composites increase significantly with increasing $\mathrm{SA} / \mathrm{V}$ and the prismatic aggregate decreases significantly with increasing SA/V. With the same SA/V the behavior of spherical aggregate is similar to the cylindrical one and they result in lower \% Reversibility. It is also observed that the effect of changing aggregate size is stronger in the HSC types.

The stress concentration and microcracking within the ITZ can be defined on the basis of the events that could occur around the aggregate depending on its surface properties. While good mechanical interlock contributes to better bond properties and therefore hinders the microcracking growth and propagation, extremes in aggregate shape may lead to higher internal stress concentrations and hence easier bond failure.

In this investigation, among the three different aggregate shapes, the prismatic shape can provide the highest mechanical interlock and least uniform stress concentration than the other two. The spherical shape provides the most uniform stress distribution around the aggregate. Cylindrical aggregates, on the other hand, provide higher mechanical interlock than the spherical aggregates but as far as stress concentration is concerned they provide a more uniform stress distribution than the prismatic aggregates. 


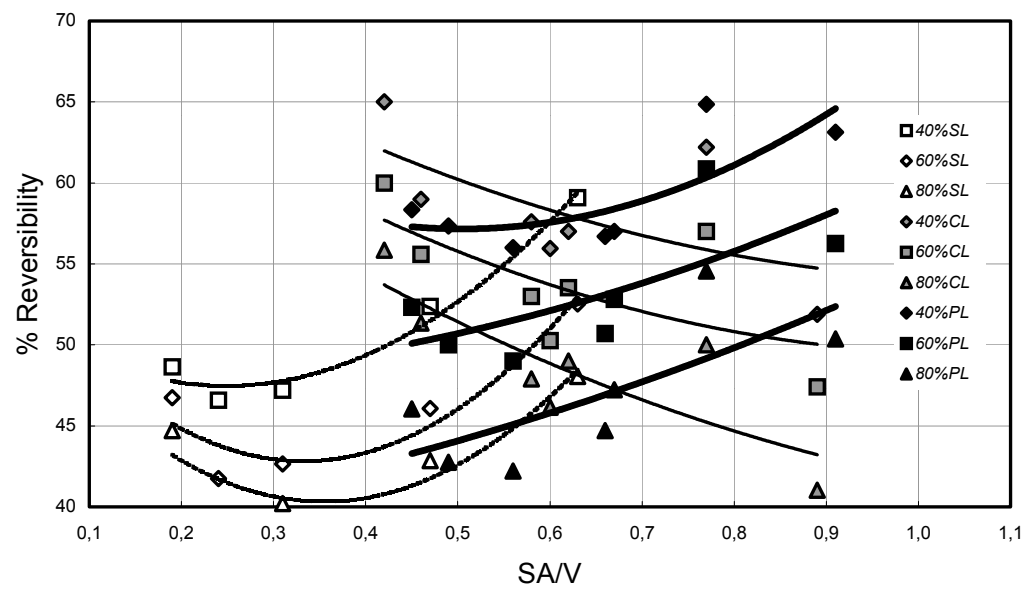

Figure 1: Effect of SA/V of spherical, cylindrical and prismatic aggregate on \% Reversibility of LSC.

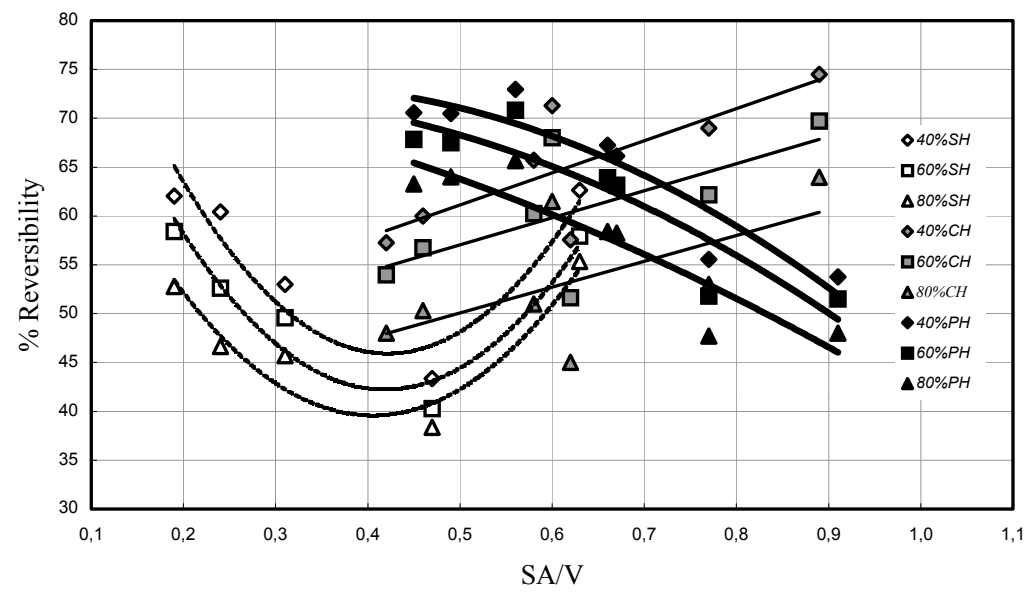

Figure 2: Effect of SA/V of spherical, cylindrical and prismatic aggregate on \% Reversibility of HSC

In order to better emphasize the shape effect of the aggregate, on the \% Reversibility, it was plotted as a function of the Length/Width (L/D) ratio of the aggregates. Figs (3) and (4) show the intercomparison between the prismatic and cylindrical aggregate shapes for HSC and LSC respectively. At L/D ratios around or equal to 1 (equidimensionality) the \% Reversibility of both cylindrical and prismatic aggregate shapes are about equal. For HSC with increasing L/D ratio the $\%$ Reversibility of the prismatic shapes increase and the cylindrical shapes decrease. The LSC shows an opposite behavior beyond L/D ratio of 1 . However, the changes are not as significant as in the case of HSC. 


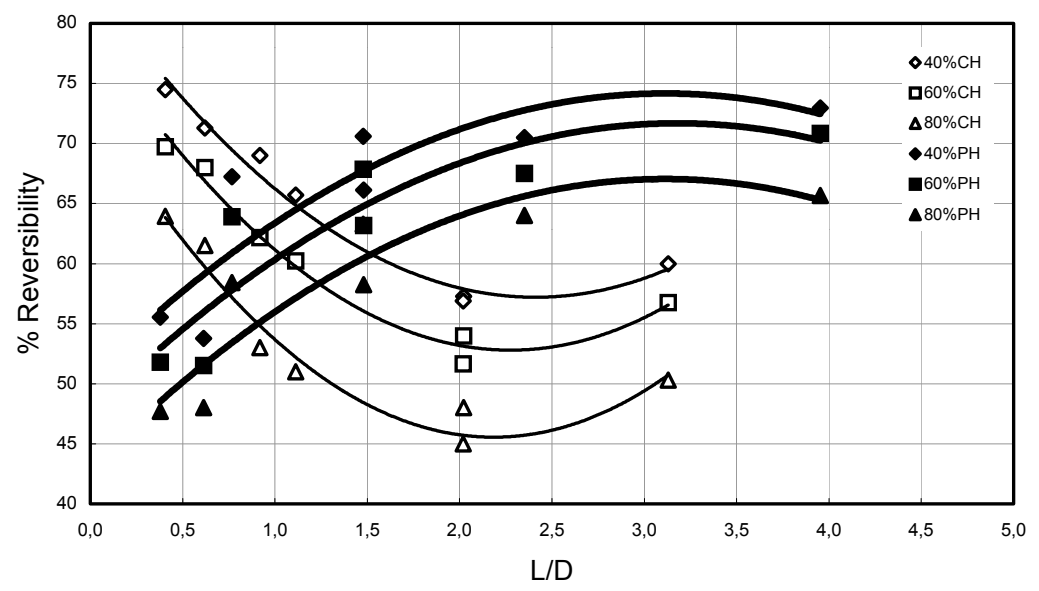

Figure 3: Effect of L/D ratio of cylindrical and prismatic aggregate on \% Reversibility of HSC.

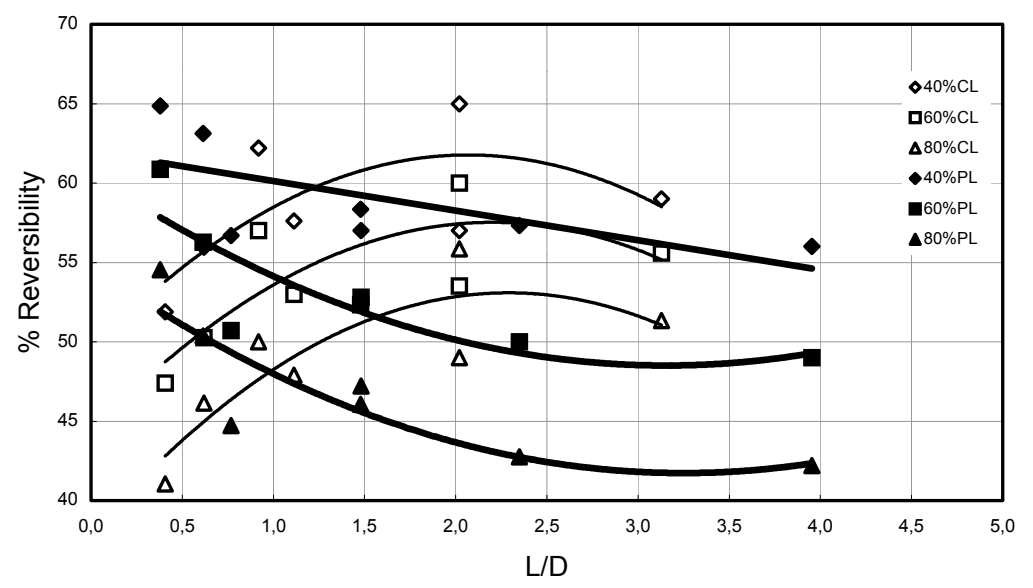

Figure 4: Effect of L/D ratio of cylindrical and prismatic aggregate on \% Reversibility of LSC.

In HSC, the positive effect of mechanical interlock is more dominant than the negative effect due to sharp edges on microcracking behavior of ITZ and therefore the composite. This effect increases with increasing L/D ratio (aggregate size). As it can be observed from Fig. (3) \% Reversibility in HSC containing prismatic aggregates, increases with increasing L/D ratio (aggregate size). This is because of increased mechanical interlock and decreased edge effect of the prismatic aggregate on microcracking process. In an opposite manner \% Reversibility in HSC composites containing cylindrical aggregate, decreases with increasing $\mathrm{L} / \mathrm{D}$ ratio. This is due to lower mechanical interlock and less significant stress concentration for large $\mathrm{L} / \mathrm{D}$ ratios. On the other hand the cylindrical aggregates with $\mathrm{L} / \mathrm{D}$ ratio less than 1, result in a lower damage than the prismatic aggregates. This is because, with the decreasing $\mathrm{L} / \mathrm{D}$ ratio, the stress concentration 
becomes more dominant and the mechanical interlocking becomes less significant. At around $\mathrm{L} / \mathrm{D}=1$ both the prismatic and cylindrical shapes result in similar damage. This is due to the equidimensionality of the aggregate. At this L/D ratio the positive and the negative effects of mechanical interlock and stress concentrations compensate each other.

In LSC (Fig (4)) with increased w/c ratio, the effect of the aggregate properties (mechanical or physical) becomes less pronounced and thus the damage process is mostly controlled by the matrix. There are only slight differences in the \% Reversibility of the cylindrical and prismatic composites. For large L/D ratios, the higher the \% Reversibility of the cylindrical shape is due to a more uniform stress concentration than the prismatic shape. With small L/D ratios and small surface areas of the aggregates, the higher mechanical interlock property of the prismatic shapes becomes more effective and increases the \% Reversibility, such that it becomes slightly higher than the cylindrical.

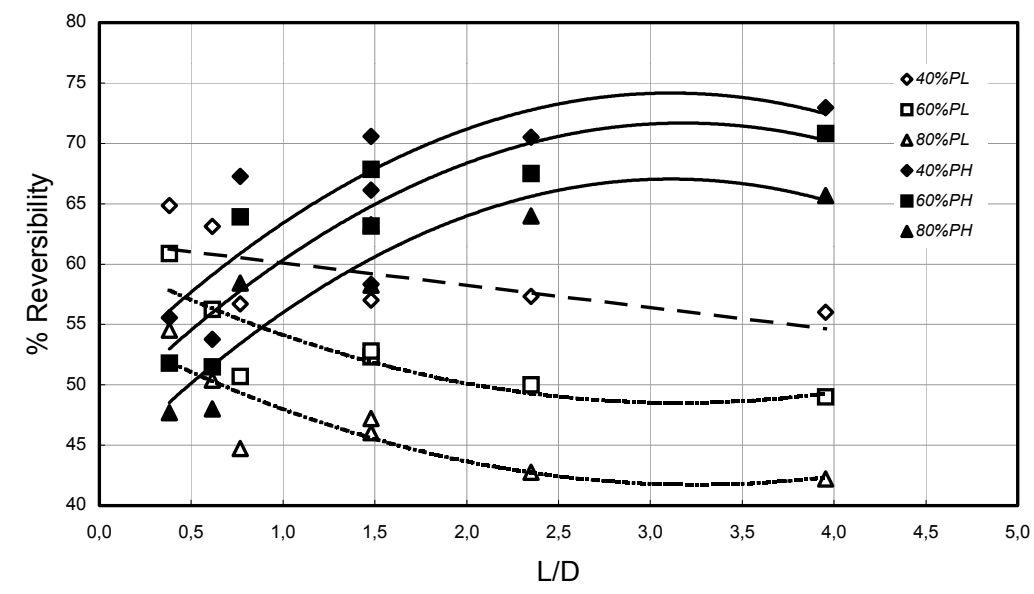

Figure 5: Effect of L/D ratio of prismatic aggregate on \% Reversibility of LSC and HSC

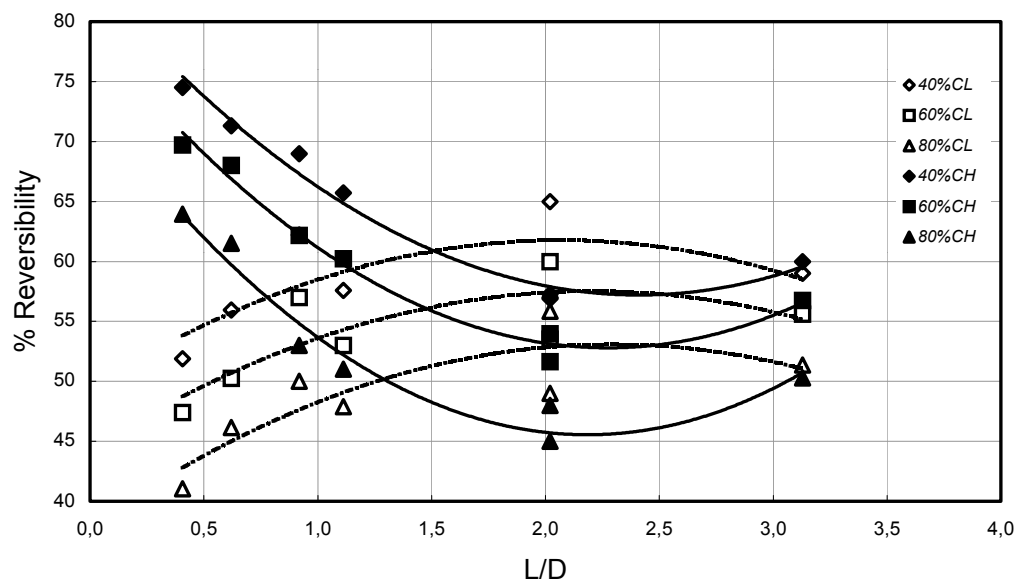

Figure 6: Effect of L/D ratio of cylindrical aggregate on \% Reversibility of LSC and HSC. 
Figs. (5) and (6) show the \% Reversibility as a function of L/D ratio for the prismatic and cylindrical shaped aggregates respectively and for LSC and HSC. In the composites that have large L/D ratios cylindrical aggregates (Fig. (6)) the LSC and HSC resemble each other. However, for smaller L/D values the HSC give higher \% Reversibility. For the prismatic aggregate however the $\%$ Reversibility of HSC for L/D ratio $\geq 1$ is much higher from that of LSC. For L/D ratio that is less than 1 , the $\%$ Reversibility values are similar.

As concluded earlier, for HSC with large aggregate sizes, the mechanical interlock is more dominant than the stress concentrations. For LSC with similarly sized aggregates, the stress concentrations are more dominant than the mechanical interlock. Thus, as it can be observed in Fig. (5), higher \% Reversibility is obtained for HSC containing primatic aggregate. As the aggregate size is decreased, the stress concentrations become more dominant for HSC, and mechanical interlock becomes more dominant for LSC. Thus, the \% Reversibility of HSC and LSC becomes approximately equal.

The cylindrical aggregates have more uniform stress distribution and worse mechanical interlock properties when compared with the prismatic aggregates. The microcracking behaviour of composites containing cylindrical aggregate is shown in Fig (6) for LSC and HSC. For large aggregates \% Reversibility in HSC goes down due to worse mechanical interlock. For the same size aggregates and in LSC, \% Reversibility goes up due to lower stress concentrations. Thus, \% Reversibility for both LSC and HSC, incorporated with large cylindrical aggregates, are about the same. For small aggregates (L/D less than 1) \% Reversibility in HSC increases due a more dominant stress concentration, and in LSC, decreases due a more dominant mechanical interlock property.

\section{CONCLUSIONS}

The degree of mechanical interlocking and internal stress concentration around the aggregate, related to the aggregate surface characteristics, are found to have significant influence on microcracking behaviour of concrete composites. Furthermore, aggregate surface characteristics become more pronounced with increased matrix quality. For HSC (low w/c) with large aggregate, the degree of mechanical interlock is more dominant than the uniformity in stress concentration. For LSC (high w/c) with large aggregate, uniformity in stress concentration is more significant than the degree of mechanical interlock.

\section{REFERENCES}

[1] Shah, S.P., and Chandra, S., Critical Stress, Volume Change and Microcracking of Concrete, ACI Materials Journal, Vol 65 (9), 770-781, 1968.

[2] Mitsuru, s., and Mitsunori, K., Resistance of the Cement-Aggregate Interfacial Zone to the Propagation of Cracks, Cement and Concrete Research, Vol 16, 653-661, 1986.

[3] Jones, K. And Kaplan, M.F., The Effects of Coarse Aggregate on the mode of Failure of Concrete in Compression and Flexure, Magazine of Concrete Research, Vol 9 (26), 89-94, 1957.

[4] Chiaia B., van Mier J.G.M., and Vervuurt, A., Crack Growth Mechanisms in Four Different Concretes: Microscopic Observations and Fractal Analyses, Cement and Concrete Research, Vol 28, 103-114, 1998.

[5] Prokopski, G., and Halbiniak, J., Interfacial Transition Zone in Cementitious Materials, Cement and Concrete Research, Vol 30, 579-583, 2000.

[6] Bascoul, A., and Maso, J.C., Influence of Intermediate Stress on the Mechanical Behavior of Concrete under Biaxial Compression, Materials and Buildings Research, Annales ITBTP, No. 351, 1977.

[7] Ringot, E., Development of the Map Cracking in Concrete under Compressive Loading, Cement and Concrete Research, Vol 18, 933-942, 1988. 